\title{
Bilateral Variations and Sexual Dimorphism in Morphometric Parameters of Infraorbital Groove, Canal and Foramen in Human Foetuses
}

\author{
Variaciones Bilaterales y Dimorfismo Sexual en los Parámetros Morfométricos \\ del Surco, Canal y Foramen Infraorbitario en Fetos Humanos
}

"Farah Ghaus \& **Faruqi N. A.

GHAUS, F. \& FARUQI, N. A. Bilateral variations and sexual dimorphism in morphometric parameters of infraorbital groove, canal and foramen in human foetuses. Int. J. Morphol., 27(2):475-480, 2009.

SUMMARY: Foetal anatomy seems to be highly promising developing speciality in the recent past. Maxilla is the key to facial skeleton. Its anatomy in general and bilateral variations along with sexual dimorphism in particular are of great surgical and forensic importance. Thirty two maxillae of sixteen human foetuses ( $21 \mathrm{wks}$ to $34 \mathrm{wks}$ ) were considered to measure lengths of infraorbital groove and canal, widths of ends of infraorbital groove, diameters of infraorbital foramen and the distances of latter from infraorbital margin and nasal notch. Groups I (21-25 wks) and II (26-30 wks) foetuses were crucial for bilateral variations for most of the parameters. Distance between infraorbital foramen and nasal notch showed variations on two sides in maximum foetal groups. Infraorbital canal was found to be larger in groups II and III foetuses in females. Width of the posterior end of infraorbital groove was less in group I, equal in group II and more in group III in males. Sexual dimorphism was noticed in all the groups for the distance of infraorbital foramen from infraorbital margin. Distance of infraorbital foramen from nasal notch did not show sexual dimorphism in most of the groups except group I where value was more in males. Rule of generalized phenomenon of larger skeleton in male was not applicable in most of the groups.

KEY WORDS: Bilateral variation; Sexual dimorphism; Infraorbital groove; Infraorbital canal; Infraorbital foramen; Human foetuses.

\section{INTRODUCTION}

Infraorbital groove, canal and foramen are important landmarks in the floor of orbit for the passage of infraorbital nerve.Blocking of latter to anaesthetize the upper jaw is oftenly done for surgical purposes in adults. This needs the accurate knowledge of the aforementioned landmarks. Enormous literature is available providing informations about these structures in adult (Kazkayasi et al., 2001, 2003; Hwang \& Baik, 1999; Lee et al., 2006; Elias et al., 2004; Hindy \& Abdel-Raouf, 1993). Recently the various invasive and noninvasive approaches aimed at correction of different foetal defects had led to the emergence of a highly promising superspeciality of foetal therapy. Foetal repair of the congenital diaphragmatic hernia was technically made possible by Soper (1984). Establishment of the correlation between location of the anterior end of infraorbital foramen and cleft palate by McKinstry (1987) has further enhanced the scope of study on foetal anatomy.Some information about the morphometry of foetal maxillary anatomy is available in literature (Ghaus \& Faruqi, 2006, 2007) but bilateral variations and sexual dimorphism of foetal infraorbital groove, canal and foramen are lacking although such records will be of great forensic and surgical importance.

\section{MATERIAL AND METHOD}

Sixteen human foetuses (12 males and 4 females) whose age ranged from 21 to $34 \mathrm{wk}$, were obtained from the museum of the anatomy department of J. N. Medical College, A. M. U., Aligarh. Maxillae of these foetuses were dissected and removed. Specimens were divided into three groups (I,

* Lecturer, MBBS, MD. Department of Anatomy, J N Medical College, Aligarh Muslim University, Aligarh, India.
** Professor, MBBS, MS. Department of Anatomy, J N Medical College, Aligarh Muslim University, Aligarh, India. 
II and III) according to ages of foetuses which ranged from 21-25 wks, 26-30 wks and more than 30 wks respectively. Periosteum and soft tissues were carefully removed under dissecting microscope to clean the maxillae from all the sides (Figs.1 and 2). Infraorbital groove, canal and foramen were measured with the help of Vernier callipers.

Lengths of infraorbital canal and groove, widths of latter at two extremes, diameters (transverse and vertical) of infraorbital foramen and its distance from infraorbital margin and nasal notch were measured by using Vernier callipers. Values were divided into three groups on the line of subgrouping of foetuses according to the age (Table I and II). Measurements of aforementioned eight parameters were presented in tabulated form (Table III-XVIII). Student's ' $t$ ' test was used for statistical analysis of results.

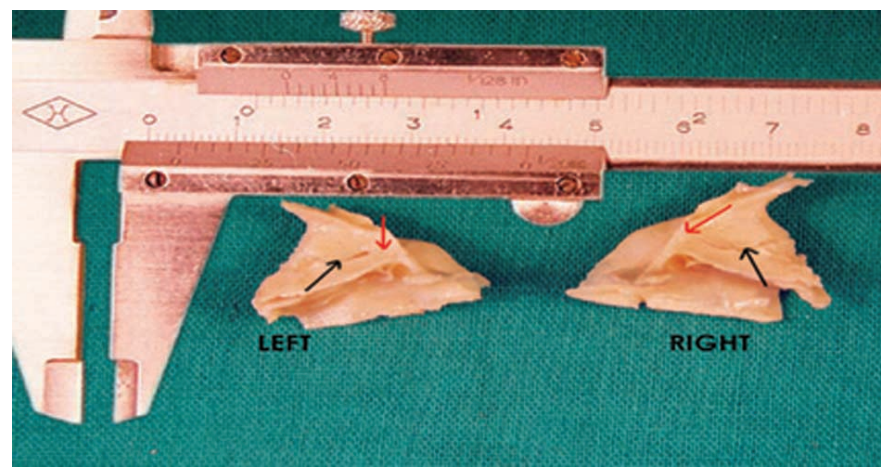

Fig. 1. Right and left maxillae showing infraorbital groove $(\rightarrow)$ and canal $(\rightarrow)$ in the orbital surface.

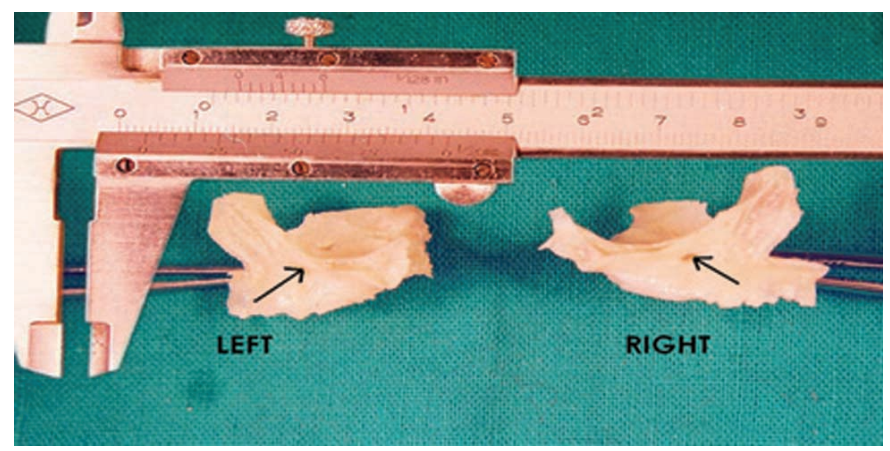

Fig. 2. Right and left maxillae showing the infraorbital foramen $(\rightarrow)$ on the anterior surface

\section{RESULTS AND DISCUSSION}

Length of infraorbital groove (Table IV) and width of anterior end of infraorbital groove (Table V) are devoid of bilateral variations. It is interesting to note that the length of infraorbital canal (Table III) and width of posterior end of infraorbital groove (Table VI) show bilateral variations only in group I with preferential increase in values on left side. On the other hand,readings pertaining to infraorbital foramen present with bilateral variations invariably in group II without any preference to side(Table VII, VIII, IX and X) with additional variations also noticed for distance between infraorbital foramen and infraorbital margin in group III (Table IX) and distance between infraorbital foramen and nasal notch in group I (Table X). Results indicate that bilateral variations maximally affect readings associated with infraorbital foramen.Such informations are lacking specifically in literature but the spurt of growth in foetuses of groups I and II for some parameters are earlier reported (Ghaus \& Faruqi, 2006; Khan \& Faruqi, 2006).

Availability of very few female foetuses as compared to male ones and reduction in number of readings in some materials due to technical reasons had posed some difficulty in analysis for sexual dimorphism results.Enormous literature are available showing sexing of infraorbital canal and foramen in adult human bones (Lee et al.; Aziz et al., 2000). Ghaus \& Faruqi (2006) showed sexual dimorphism for the first time in human foetal maxillary sinuses. In our study, infraorbital canal was found to be larger in groups II and III foetuses in females (Table XI). The values of the length of infraorbital groove (Table XII) and its width at the anterior end (Table XIII) and vertical diameter of infraorbital foramen (Table XVI) showed no differences in two sexes. All the other parameters i.e. length of infraorbital canal (Table XI), width of posterior end of infraorbital groove (Table XIV), transverse diameter of infraorbital foramen (Table XV) and distance of infraorbital foramen from infraorbital margin and nasal notch (Tables XVII and XVIII ) present with sexual dimorphism. Surprisingly in most of the cases, values are more in females, as supported by statistical analysis. Another striking feature is the involvement of last group in

Table I. Subgrouping of foetal maxillae of two sides.

\begin{tabular}{lccccc}
\hline Groups & Age (wks) & $\begin{array}{l}\mathbf{N}^{\mathbf{0}} \text { right } \\
\text { maxillae }\end{array}$ & $\mathbf{N}^{\mathbf{0}}$ left maxillae & Total $\mathbf{N}^{\mathbf{0}}$ of maxillae \\
\hline I & $21-25$ & 8 & 8 & 16 \\
II & $26-30$ & 5 & 5 & 10 \\
III & $>30$ & 3 & 3 & 06 \\
\hline
\end{tabular}


majority. Ghaus \& Faruqi (2006) reported larger anteroposterior and transverse diameters of maxillary sinus in males in group II and I foetuses respectively. Sexual dimorphism in maxillary sinus was also noticed by
Arredondo de Arreola et al. (1996) in human embryo and Koppe et al. (2000) in monkey. Infraorbital groove, canal and foramen, developing in close proximity to maxillary sinus are, therefore, expected to show sexual dimorphism.

Table II. Subgrouping of foetal maxillae in two sexes.

\begin{tabular}{lcccc}
\hline Groups & $\begin{array}{c}\text { Age } \\
\text { (wks) }\end{array}$ & $\begin{array}{c}\mathbf{N}^{\mathbf{0}} \text { maxillae from } \\
\text { male foetuses }\end{array}$ & $\begin{array}{c}\mathbf{N}^{\mathbf{0}} \text { maxillae from } \\
\text { female foetuses }\end{array}$ & $\begin{array}{c}\text { Total } \mathbf{N}^{\mathbf{0}} \\
\text { of maxillae }\end{array}$ \\
\hline I & $21-25$ & 14 & 2 & 16 \\
II & $26-30$ & 6 & 4 & 10 \\
III & $>30$ & 4 & 2 & 6 \\
\hline
\end{tabular}

Table III. Length of infraorbital canal of two sides in human foetuses.

\begin{tabular}{lcccccc}
\hline Groups & \multicolumn{2}{c}{ Right side } & \multicolumn{2}{c}{ Left side } & $\begin{array}{c}\text { Percent } \\
\text { difference }\end{array}$ & P Value \\
\cline { 2 - 5 } & $\mathbf{N}^{\mathbf{0}}$ of cases & Mean $\pm \mathbf{S D}(\mathbf{m m})$ & $\mathbf{N}^{\mathbf{0}}$ of cases & Mean $\pm \mathbf{S D}(\mathbf{m m})$ & & \\
\hline I & 4 & $1.05 \pm 0.41$ & 4 & $1.49 \pm 0.49$ & 30 & $<0.05$ \\
II & 5 & $3.21 \pm 2.00$ & 3 & $3.18 \pm 2.81$ & 01 & $<0.8$ \\
III & 3 & $2.40 \pm 0.70$ & 3 & $2.85 \pm 0.59$ & 16 & $<0.1$ \\
\hline
\end{tabular}

Table IV. Length of infraorbital groove of two sides in human foetuses.

\begin{tabular}{|c|c|c|c|c|c|c|}
\hline \multirow[t]{2}{*}{ Groups } & \multicolumn{2}{|c|}{ Right side } & \multicolumn{2}{|c|}{ Left side } & \multirow{2}{*}{$\begin{array}{c}\text { Percent } \\
\text { difference }\end{array}$} & \multirow[t]{2}{*}{ P Value } \\
\hline & $\mathbf{N}^{0}$ of cases & Mean \pm SD $(\mathbf{m m})$ & $\mathrm{N}^{\circ}$ of cases & Mean \pm SD $(\mathbf{m m})$ & & \\
\hline I & 8 & $4.58 \pm 0.90$ & 8 & $5.38 \pm 1.62$ & 15 & $<0.1$ \\
\hline II & 5 & $5.54 \pm 1.43$ & 5 & $6.29 \pm 2.27$ & 12 & $<0.2$ \\
\hline III & 3 & $5.90 \pm 1.89$ & 3 & $6.12 \pm 1.36$ & 4 & $<0.5$ \\
\hline
\end{tabular}

Table V. Width of the anterior end of infraorbital groove of two sides in human foetuses.

\begin{tabular}{|c|c|c|c|c|c|c|}
\hline \multirow[t]{2}{*}{ Groups } & \multicolumn{2}{|c|}{ Right side } & \multicolumn{2}{|c|}{ Left side } & \multirow{2}{*}{$\begin{array}{c}\text { Percent } \\
\text { difference }\end{array}$} & \multirow[t]{2}{*}{ P Value } \\
\hline & $\mathbf{N}^{0}$ of cases & Mean \pm SD $(\mathrm{mm})$ & $N^{0}$ of cases & Mean \pm SD $(\mathrm{mm})$ & & \\
\hline $\mathbf{I}$ & 8 & $1.20 \pm 0.50$ & 8 & $1.43 \pm 0.51$ & 16 & $<0.8$ \\
\hline II & 5 & $1.42 \pm 0.34$ & 5 & $1.48 \pm 0.46$ & 04 & $<0.8$ \\
\hline III & 3 & $1.55 \pm 0.22$ & 3 & $1.78 \pm 0.33$ & 15 & $<0.1$ \\
\hline
\end{tabular}

Table VI. Width of the posterior end of infraorbital groove of two sides in human foetuses.

\begin{tabular}{|c|c|c|c|c|c|c|}
\hline \multirow[t]{2}{*}{ Groups } & \multicolumn{2}{|c|}{ Right side } & \multicolumn{2}{|c|}{ Left side } & \multirow{2}{*}{$\begin{array}{l}\text { Per cent } \\
\text { difference }\end{array}$} & \multirow[t]{2}{*}{ P Value } \\
\hline & $\mathrm{N}^{\circ}$ of cases & Mean \pm SD $(\mathrm{mm})$ & $N^{0}$ of cases & Mean \pm SD $(\mathrm{mm})$ & & \\
\hline I & 8 & $1.85 \pm 0.49$ & 8 & $2.19 \pm 0.46$ & 16 & $<0.001$ \\
\hline II & 5 & $2.59 \pm 0.21$ & 5 & $2.71 \pm 0.39$ & 04 & $<0.2$ \\
\hline III & 3 & $3.03 \pm 0.45$ & 3 & $3.00 \pm 0.36$ & 1 & $<0.8$ \\
\hline
\end{tabular}


GHAUS, F. \& FARUQI, N. A. Bilateral variations and sexual dimorphism in morphometric parameters of infraorbital groove, canal and foramen in human foetuses. Int. J. Morphol., 27(2):475-480, 2009.

Table VII. Transverse diameter of infraorbital foramen of two sides in human foetuses.

\begin{tabular}{|c|c|c|c|c|c|c|}
\hline \multirow[t]{2}{*}{ Groups } & \multicolumn{2}{|c|}{ Right side } & \multicolumn{2}{|c|}{ Left side } & \multirow{2}{*}{$\begin{array}{c}\text { Percent } \\
\text { difference }\end{array}$} & \multirow[t]{2}{*}{ P Value } \\
\hline & $\mathrm{N}^{\circ}$ of cases & Mean \pm SD $(\mathrm{mm})$ & $N^{0}$ of cases & Mean \pm SD $(\mathrm{mm})$ & & \\
\hline I & 4 & $1.15 \pm 0.24$ & 3 & $1.15 \pm 0.43$ & 00 & $<0.8$ \\
\hline II & 5 & $2.30 \pm 0.35$ & 4 & $1.93 \pm 0.28$ & 19 & $<0.002$ \\
\hline III & 3 & $2.02 \pm 0.57$ & 3 & $1.85 \pm 0.66$ & 09 & $<0.2$ \\
\hline
\end{tabular}

Table VIII. Vertical diameter of infraorbital foramen of two sides in human foetuses.

\begin{tabular}{|c|c|c|c|c|c|c|}
\hline \multirow{2}{*}{ Groups } & \multicolumn{2}{|c|}{ Right side } & \multicolumn{2}{|c|}{ Left side } & \multirow{2}{*}{$\begin{array}{l}\text { Per cent } \\
\text { difference }\end{array}$} & \multirow[t]{2}{*}{ P Value } \\
\hline & $\mathbf{N}^{0}$ of cases & Mean \pm SD $(\mathrm{mm})$ & $N^{\circ}$ of cases & Mean \pm SD $(\mathrm{mm})$ & & \\
\hline I & 4 & $0.83 \pm 0.16$ & 3 & $0.80 \pm 0.23$ & 04 & $<0.5$ \\
\hline II & 5 & $1.12 \pm 0.14$ & 4 & $1.20 \pm 0.22$ & 07 & $<0.05$ \\
\hline III & 3 & $1.18 \pm 0.19$ & 3 & $1.25 \pm 0.001$ & 06 & $<0.8$ \\
\hline
\end{tabular}

Table IX. Distance of infraorbital foramen from infraorbital margin on the two sides in human foetuses.

\begin{tabular}{|c|c|c|c|c|c|c|}
\hline \multirow[t]{2}{*}{ Groups } & \multicolumn{2}{|c|}{ Right side } & \multicolumn{2}{|c|}{ Left side } & \multirow{2}{*}{$\begin{array}{c}\text { Percent } \\
\text { difference }\end{array}$} & \multirow[t]{2}{*}{ P Value } \\
\hline & $\mathrm{N}^{0}$ of cases & Mean \pm SD $(\mathrm{mm})$ & $\mathrm{N}^{\circ}$ of cases & Mean \pm SD $(\mathrm{mm})$ & & \\
\hline I & 8 & $1.34 \pm 0.27$ & 7 & $1.37 \pm 0.35$ & 02 & $<0.5$ \\
\hline II & 5 & $1.40 \pm 0.34$ & 5 & $1.69 \pm 0.47$ & 17 & $<0.05$ \\
\hline III & 3 & $1.75 \pm 0.25$ & 3 & $2.27 \pm 0.34$ & 23 & $<0.01$ \\
\hline
\end{tabular}

Table X. Distance of infraorbital foramen from nasal notch on the two sides in human foetuses.

\begin{tabular}{|c|c|c|c|c|c|c|}
\hline \multirow[t]{2}{*}{ Groups } & \multicolumn{2}{|c|}{ Right side } & \multicolumn{2}{|c|}{ Left side } & \multirow{2}{*}{$\begin{array}{l}\text { Per cent } \\
\text { difference }\end{array}$} & \multirow[t]{2}{*}{ P Value } \\
\hline & $\mathrm{N}^{0}$ of cases & Mean \pm SD $(\mathrm{mm})$ & $\mathrm{N}^{\circ}$ of cases & Mean \pm SD $(\mathrm{mm})$ & & \\
\hline $\mathbf{I}$ & 8 & $4.91 \pm 1.00$ & 8 & $4.60 \pm 1.06$ & 07 & $<0.02$ \\
\hline II & 5 & $5.86 \pm 0.81$ & 5 & $6.04 \pm 0.82$ & 04 & $<0.05$ \\
\hline III & 3 & $5.83 \pm 1.39$ & 3 & $6.32 \pm 0.93$ & 08 & $<0.2$ \\
\hline
\end{tabular}

Table XI. Length of infraorbital canal of human foetuses in two sexes.

\begin{tabular}{|c|c|c|c|c|c|c|}
\hline \multirow{2}{*}{ Groups } & \multicolumn{2}{|c|}{ Male foetuses } & \multicolumn{2}{|c|}{ Female foetuses } & \multirow{2}{*}{$\begin{array}{c}\text { Percent } \\
\text { difference }\end{array}$} & \multirow[t]{2}{*}{ P Value } \\
\hline & $N^{0}$ of cases & Mean \pm SD $(\mathrm{mm})$ & $\mathbf{N}^{0}$ of cases & Mean \pm SD $(\mathrm{mm})$ & & \\
\hline I & 6 & $1.31 \pm 0.53$ & 2 & $1 \pm 0.001$ & 24 & $<0.2$ \\
\hline II & 5 & $1.79 \pm 0.94$ & 3 & $5.55 \pm 2.05$ & 210 & $<0.01$ \\
\hline III & 4 & $2.21 \pm 0.43$ & 2 & $3.45 \pm 0.15$ & 56 & $<0.002$ \\
\hline
\end{tabular}

Table XII. Length of infraorbital groove of human foetuses in two sexes.

\begin{tabular}{|c|c|c|c|c|c|c|}
\hline \multirow[t]{2}{*}{ Groups } & \multicolumn{2}{|c|}{ Male foetuses } & \multicolumn{2}{|c|}{ Female foetuses } & \multirow{3}{*}{$\begin{array}{c}\text { Percent } \\
\text { difference } \\
7\end{array}$} & \multirow[t]{2}{*}{ P Value } \\
\hline & $\mathrm{N}^{\circ}$ of cases & Mean \pm SD $(\mathrm{mm})$ & $N^{\circ}$ of cases & Mean \pm SD $(\mathrm{mm})$ & & \\
\hline I & 14 & $4.98 \pm 1.57$ & 2 & $4.65 \pm 0.05$ & & $<0.2$ \\
\hline II & 6 & $6.83 \pm 1.40$ & 4 & $5.16 \pm 2.31$ & 24 & $<0.5$ \\
\hline III & 4 & $6.81 \pm 1.24$ & 2 & $4.40 \pm 1.10$ & 35 & $<0.2$ \\
\hline
\end{tabular}


GHAUS, F. \& FARUQI, N. A. Bilateral variations and sexual dimorphism in morphometric parameters of infraorbital groove, canal and foramen in human foetuses. Int. J. Morphol., 27(2):475-480, 2009.

Table XIII. Width of the anterior end of infraorbital groove of human foetuses in two sexes.

\begin{tabular}{lcccccc}
\hline Groups & \multicolumn{2}{c}{ Male foetuses } & \multicolumn{2}{c}{ Female foetuses } & PerTabla & P Value \\
\cline { 2 - 5 } & $\mathbf{N}^{\mathbf{0}}$ of cases & Mean \pm SD $(\mathbf{m m})$ & $\mathbf{N}^{\mathbf{0}}$ of cases & Mean \pm SD $(\mathbf{m m})$ & XIII & \\
\hline I & 14 & $1.33 \pm 0.55$ & 2 & $1.23 \pm 0.03$ & 8 & $<0.5$ \\
II & 6 & $1.43 \pm 0.44$ & 4 & $3.54 \pm 0.47$ & 8 & $<0.2$ \\
III & 4 & $1.55 \pm 0.23$ & 2 & $1.85 \pm 0.35$ & 19 & $<0.2$ \\
\hline
\end{tabular}

Table XIV. Width of the posterior end of infraorbital groove of human foetuses in two sexes.

\begin{tabular}{|c|c|c|c|c|c|c|}
\hline \multirow[t]{2}{*}{ Groups } & \multicolumn{2}{|c|}{ Male foetuses } & \multicolumn{2}{|c|}{ Female foetuses } & \multirow{2}{*}{$\begin{array}{l}\text { Per cent } \\
\text { difference }\end{array}$} & \multirow[t]{2}{*}{ P Value } \\
\hline & $\mathrm{N}^{0}$ of cases & Mean \pm SD $(\mathrm{mm})$ & $\mathrm{N}^{0}$ of cases & Mean \pm SD $(\mathrm{mm})$ & & \\
\hline I & 14 & $1.94 \pm 0.50$ & 2 & $2.55 \pm 0.05$ & 31 & $<0.001$ \\
\hline II & 6 & $2.77 \pm 0.21$ & 4 & $2.48 \pm 0.37$ & 10 & $<0.1$ \\
\hline III & 4 & $3.11 \pm 0.41$ & 2 & $2.83 \pm 0.33$ & 9 & $<0.02$ \\
\hline
\end{tabular}

Table XV. Transverse diameter of infraorbital foramen of human foetuses in two sexes.

\begin{tabular}{|c|c|c|c|c|c|c|}
\hline \multirow[t]{2}{*}{ Groups } & \multicolumn{2}{|c|}{ Male foetuses } & \multicolumn{2}{|c|}{ Female foetuses } & \multirow{2}{*}{$\begin{array}{l}\text { Percent } \\
\text { difference }\end{array}$} & \multirow[t]{2}{*}{ P Value } \\
\hline & $\mathbf{N}^{0}$ of cases & Mean \pm SD $(\mathrm{mm})$ & $\mathrm{N}^{\mathbf{0}}$ of cases & $\operatorname{Mean} \pm$ SD $(\mathrm{mm})$ & & \\
\hline II & 6 & $2.25 \pm 0.39$ & 3 & $2.10 \pm 0.001$ & 7 & $<0.2$ \\
\hline III & 4 & $1.74 \pm 0.63$ & 2 & $2.33 \pm 0.38$ & 34 & $<0.02$ \\
\hline
\end{tabular}

Table XVI. Vertical diameter of infraorbital foramen of human foetuses in two sexes.

\begin{tabular}{|c|c|c|c|c|c|c|}
\hline \multirow[t]{2}{*}{ Groups } & \multicolumn{2}{|c|}{ Male foetuses } & \multicolumn{2}{|c|}{ Female foetuses } & \multirow{2}{*}{$\begin{array}{c}\text { Percent } \\
\text { difference }\end{array}$} & \multirow[t]{2}{*}{ P Value } \\
\hline & $N^{0}$ of cases & Mean \pm SD $(\mathrm{mm})$ & $N^{0}$ of cases & Mean \pm SD $(\mathrm{mm})$ & & \\
\hline II & 6 & $1.18 \pm 0.21$ & 3 & $1.10 \pm 0.08$ & 7 & $<0.2$ \\
\hline III & 4 & $1.24 \pm 0.16$ & 2 & $1.18 \pm 0.08$ & 5 & $<0.2$ \\
\hline
\end{tabular}

Table XVII. Distance of infraorbital foramen from infraorbital margin of human foetuses in two sexes.

\begin{tabular}{|c|c|c|c|c|c|c|}
\hline \multirow[t]{2}{*}{ Groups } & \multicolumn{2}{|c|}{ Male foetuses } & \multicolumn{2}{|c|}{ Female foetuses } & \multirow{2}{*}{$\begin{array}{c}\text { Percent } \\
\text { difference }\end{array}$} & \multirow[t]{2}{*}{ P Value } \\
\hline & $N^{\circ}$ of cases & Mean \pm SD $(\mathrm{mm})$ & $N^{\circ}$ of cases & Mean \pm SD $(\mathrm{mm})$ & & \\
\hline I & 13 & $1.29 \pm 0.27$ & 2 & $1.80 \pm 0.001$ & 40 & $<0.001$ \\
\hline II & 6 & $1.46 \pm 0.47$ & 4 & $1.68 \pm 0.34$ & 15 & $<0.02$ \\
\hline III & 4 & $1.83 \pm 0.23$ & 2 & $2.43 \pm 0.33$ & 35 & $<0.02$ \\
\hline
\end{tabular}

Table XVIII. Distance of infraorbital foramen from nasal notch of human foetuses in two sexes.

\begin{tabular}{|c|c|c|c|c|c|c|}
\hline \multirow[t]{2}{*}{ Groups } & \multicolumn{2}{|c|}{ Male foetuses } & \multicolumn{2}{|c|}{ Female foetuses } & \multirow{2}{*}{$\begin{array}{l}\text { Per cent } \\
\text { difference }\end{array}$} & \multirow[t]{2}{*}{ P Value } \\
\hline & $N^{0}$ of c ases & $\operatorname{Mean} \pm$ SD $(\mathrm{mm})$ & $\mathrm{N}^{0}$ of cases & Mean \pm SD $(\mathrm{mm})$ & & \\
\hline I & 14 & $4.96 \pm 0.99$ & 2 & $3.90 \pm 0.1$ & 21 & $<0.001$ \\
\hline II & 6 & $5.93 \pm 0.80$ & 4 & $5.99 \pm 0.85$ & 1 & $<0.8$ \\
\hline III & 4 & $6.48 \pm 1.34$ & 2 & $5.28 \pm 0.03$ & 19 & $<0.1$ \\
\hline
\end{tabular}


GHAUS, F. \& FARUQI, N. A. Variaciones bilaterales y dimorfismo sexual en los parámetros morfométricos del surco, canal y foramen infraorbitario en fetos humanos. Int. J. Morphol., 27(2):475-480, 2009.

RESUMEN: La anatomía fetal, con su desarrollo en los últimos años, parece ser especialidad muy prometedora. El maxilar es la clave del esqueleto facial. Su anatomía en general y las variaciones bilaterales junto al dimorfismo sexual en particular, son de gran importancia quirúrgica y forense. Fueron estudiados 32 maxilares de 16 fetos humanos ( 21 semanas a 34 semanas) en los cuales se midió la longitud del surco y canal infraorbitario, anchos de los extremos de surco infraorbitario, diámetros del foramen infraorbitario y las distancias de este último desde el margen infraorbitario hasta la escotadura nasal. Las variaciones bilaterales en la mayoría de los parámetros fue en los grupos de fetos I (21-25 semanas) y II (26-30 semanas). La distancia entre el foramen infraorbitario y escotadura nasal mostró variaciones máximas en dos partes en los grupos de fetos. El canal Infraorbitario resultó ser más largo en los grupos II y III de fetos femeninos. El ancho del extremo posterior del surco infraorbitario fue menor en el grupo de fetos masculino I, igual en el grupo II y mayor en el grupo III. El dimorfismo sexual se observó en todos los grupos en la distancia desde el foramen infraorbitario hasta el margen infraorbitario. La distancia desde el foramen infraorbitario hasta la escotadura nasal no mostró dimorfismo sexual en la mayoría de los grupos a excepción del grupo I, donde el valor fue mayor en los hombres. La norma generalizada que el esqueleto más grande es del género masculino no fue aplicable en la mayoría de los grupos.

PALABRAS CLAVE: Variación bilateral; Dimorfismo sexual; Surco infraorbitario; Canal infraorbitario; Foramen infraorbitario; Feto humano.

\section{REFERENCES}

Arredondo de Arreola, G.; López Serna, N.; de Hoyos Parra, R. $\&$ Arreola Salinas, M. A. Morphogenesis of the lateral nasal wall from 6 to 36 weeks. Otolaryngol. Head Neck Surg., 114(1):54-60, 1996.

Aziz, S. R.; Marchena, J. M. \& Puran, A. Anatomic characteristics of the infraorbital foramen: a cadaver study. J. Oral Maxillofac. Surg., 58(9):992-6, 2000.

Elias, M. G.; Silva, R. B.; Pimentel, M. L.; Cardoso, V. T. S..; Rivello, T. \& Babinski, M. A. Morphometric analysis of the infraorbital foramen and accessories foraminas in Brazilian skulls. Int. J. Morphol., 22(4):273-8, 2004.

Ghaus, F. \& Faruqi, N. A. Morphometric analysis of developing maxillary sinuses in human foetuses. Int. J. Morphol., 24(3):303-8, 2006.

Ghaus, F. \& Faruqi, N. A. Morphometric analysis of infraorbital foramen in human foetuses. Int. J. Morphol., 25(2):301-4, 2007.

Hindy, A. M. \& Abdel-Raouf, F. A study of infraorbital foramen, canal and nerve in the adult Egyptians. Egypt. Dent. J., 39(4):573-80, 1993.

Hwang, K. \& Baik, S. H. Surgical anatomy of the orbit of Korean adults. J. Craniofac. Surg., 10(2):129-34, 1999.

Kazkayasi, M.; Ergin, A.; Ersoy, M.; Bengi, O.; Tekdemir, I. \& Elhan, A. Certain anatomical relations and the precise morphometry of the infraorbital foramen-canal and groove: an anatomical and cephalometric study. Laryngoscope, 111(4 Pt 1):609-14, 2001.
Kazkayasi, M.; Ergin, A.; Ersoy, M.; Tekdemir, I. \& Elhan, A. Microscopic anatomy of the infraorbital canal, nerve, and foramen. Otolaryngol. Head Neck Surg., 129(6):692-7, 2003.

Khan, Z. \& Faruqi, N. A. Determination of gestational age of human foetuses from diaphyseal lengths of long bones $-\mathrm{A}$ radiological study. J. Anat. Society of India, 55(1):67-71, 2006.

Koppe, T.; Klauke, T.; Lee, S. H. \& Schumacher, G. Growth pattern of the maxillary sinus in the miniature pig (Sus Scrofa). Cells Tissues Organs, 167(1):58-67, 2000.

Lee, U. Y.; Nam, S. H.; Han, S. H.; Choi, K. N. \& Kim, T. J. Morphological characteristics of the infraorbital foramen and infraorbital canal using three- dimensional models. Surg. Radiol. Anat., 28(2):115-20, 2006.

McKinstry, R. E. Transverse relationships of the infraorbital foramina in cleft and noncleft individuals. Am. J. Phys. Anthropol., 74(1):109-15, 1987.

Soper, R. T.; Pringle, K. C. \& Schefeild, J. C. The creation and repair of diaphragmatic hernia in foetal lambs: techniques and survival. J. Pediatr. Surg., 19:33-40, 1984.

\section{Correspondence to:}

Dr. Ghaus Farah

Lecturer, Department of Anatomy.

Jawaharlal Nehru Medical College.

Aligarh Muslim University

Aligarh - INDIA

\section{E mail: drfarahghaus@rediffmail.com} drfarahghaus@gmail.com

Received : 18-07-2008

Accepted : 10-04-2009 